Chirurg 2010 $81: 873-874$

DOI 10.1007/s00104-010-1907-5

Online publiziert: 22. September 2010

(c) Springer-Verlag 2010

\author{
J. Weitz · M.W. Büchler \\ Chirurgische Universitätsklinik, Heidelberg
}

\title{
Onkologische Beckenchirurgie
}

logischen Tumoren des kleinen Beckens dargestellt, die sog. mesometriale Resektion (TMMR, PMMR) und die Vulvafeldresektion (VFR). Nach der Kompartimenttheorie der lokalen Tumorausbreitung ist dabei die Resektion des befallenen Kompartiments mit einer hohen lokalen $\mathrm{Tu}$ morkontrolle assoziiert, wobei durch eine Schonung anatomischer Strukturen außerhalb des Kompartiments die perioperative Morbidität minimiert werden kann. Die Darstellung der lateral erweiterten endopelvinen Resektion (LEER) bei lokal fortgeschrittenen Tumoren bzw. Rezidiven sowie der Rekonstruktionsmöglichkeiten nach Vulva- bzw. Vaginaresektion vervollständigt die ganze Palette gynäkologischer Eingriffe im Rahmen der onkologischen Beckenchirurgie.

Am Beispiel des Harnblasenkarzinoms wird die onkologische Beckenchirurgie aus urologischer Sicht beleuchtet. Neben dem Ziel, exzellente onkologische Langzeitergebnisse $\mathrm{zu}$ erreichen, muss auch die Lebensqualität der Patienten, in diesem Zusammenhang die Kontinenz und Sexualfunktion, im Fokus des operativen Eingriffs stehen. Durch eine - wo möglich - nervenschonende Operationstechnik und Ausschöpfung aller im Beitrag dargestellten Möglichkeiten der Rekonstruktion nach Zystektomie (orthotoper Blasenersatz, katheterisierbarer Pouch, Conduit) lässt sich diese Zielsetzung erreichen, wobei die individuelle Patientensituation die Wahl der Rekonstruktion bestimmt.

Bei der Darstellung der operativen Behandlung von Rektumkarzinomrezidiven gilt es zunächst hervorzuheben, dass ein ganz wesentlicher Aspekt die Reduktion des Auftretens eines Lokalrezidivs durch eine technisch adäquate Erstoperati- on ist. In dem Beitrag hierzu wird deutlich, wie anspruchsvoll die Rezidivoperation sowohl während der Resektions- als auch der Rekonstruktionsphase ist. Entsprechend ist der Eingriff mit einer hohen perioperativen Morbidität verknüpft, bietet aber die einzig kurative Option bei diesem schwierigen Patientengut. Eine besondere Herausforderung besteht auch im Hinblick auf die präoperative Diagnostik. $\mathrm{Da}$ es intraoperativ - bei den bereits voroperierten und häufig auch vorbestrahlten Patienten - extrem schwierig ist, zwischen Narbe und Tumorrezidiv zu unterscheiden und eine R1-Resektion oder eine Eröffnung des Rezidivs vermieden werden muss, sollte möglichst bereits präoperativ anhand der Bildgebung - die operative Strategie festgelegt werden.

\section{( Idealerweise sollten die Patienten im Tumorboard besprochen werden}

Die große Relevanz des interdisziplinären Vorgehens wird auch im Beitrag bezüglich der totalen Beckenexenteration deutlich. Idealerweise sollten die Patienten im Tumorboard besprochen werden, sodass alle beteiligten Fachdisziplinen frühzeitig in die Indikationsstellung und die Therapieplanung eingebunden werden, dies gilt auch für die Planung des multimodalen Therapiekonzeptes. Entsprechend erfolgt auch eine intraoperative Einbeziehung der verschiedenen Fachdisziplinen, je nach individueller Situation des Patienten. Naturgemäß sollten diese Patienten in Zentren mit entsprechender Erfahrung behandelt werden.

Neben der Auseinandersetzung mit technischen Details der onkologischen tiven Konzs 
Beckenchirurgie gilt es, die Patienten in die Entscheidungsfindung zur Operation mit einzubeziehen. Damit die Patienten in den ausgedehnten Eingriff einwilligen können, bedarf es einer präoperativen Aufklärung, die das Ausmaß des operativen Eingriffes darstellt und eine Einschätzung der postoperativen Lebensqualität erlaubt.

Wir bedanken uns bei den Autoren für die gelungenen Beiträge, die eine exzellente Übersicht über das Gebiet der onkologischen Beckenchirurgie geben.

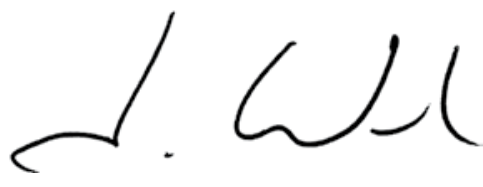

Prof. Dr. J. Weitz

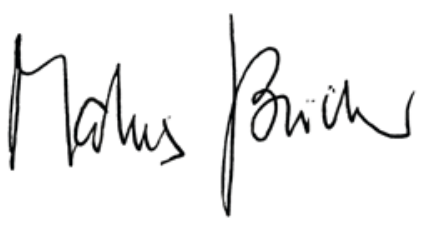

Prof. Dr. M.W. Büchler

\section{Korrespondenzadresse \\ Prof. Dr. M.W. Büchler \\ Chirurgische Universitätsklinik, Im Neuenheimer Feld 110, 69120 Heidelberg markus_buechler@med.uni-heidelberg.de}

\section{Führungskompetenzen für Leitende Ärzte im Krankenhaus}

„Es ist eine Erweiterung des Selbstverständnisses zu beobachten" - Der Autor Jens Hollmann im Interview

\section{Lange Jahre galten fast ausschließlich die medizinischen Kompetenzen als ausschlaggebend für die Besetzung ärzt- \\ ISBN: 978-3-642- \\ 05264-4 \\ $44,95 €$} licher Leitungspositionen. Gilt das aus Ihrer Sicht auch heute noch?

- Herausragende medizinische Kompetenzen sind unverändert eine Selbstverständlichkeit. Zunehmend gewinnen aber auch überfachliche Führungsqualifikationen an Bedeutung, um das Abteilungsteam bedarfsgerecht und zukunftsorientiert zu entwickeln und die Leistungsbereitschaft zu stärken. Wer sich heute um eine Position als Leitender Arzt bewirbt, muss damit rechnen, einem Auswahlverfahren unterzogen zu werden, das speziell diese Führungsqualifikationen prüft - und dies haben Ärzte weder im Studium noch in ihrer weiteren fachlichen Ausbildung erlernt.

\section{Haben sich die Herausforderungen an} die Führungskompetenz Leitender Ärzte verändert?

- Ja - und das spüren auch die meisten Chefärzte sehr deutlich. Die Jahre, in denen Assistenzärzte froh waren, eine Stelle zu bekommen und sich jeder Führung fügten, sind vorbei. Heute verzeichnen Kliniken einen Ärztemangel, und die Arbeitsatmosphäre in einer Abteilung spielt eine wichtige Rolle für die Reputation der Klinik in der Ärzterekrutierung.

Die Art der ärztlichen Führung wirkt sich also auch auf die Mitarbeiterbindung aus?

- Natürlich - und dies berührt maßgeblich die ökonomischen Interessen der Klinik. Ärztlichen Nachwuchs gezielt zu entwickeln und zu binden, ist für die Klinikleitung effektiver und effizienter als die Neugewinnung von Personal. Immer mehr Kliniken verlangen daher ein adäquates Situationsmanagement von ihren ärztlichen Führungskräften und investieren gezielt in die überfachliche Führungsausbildung der Leitenden Ärzte.

\section{Können Sie uns Beispiele nennen?}

- Die drei wichtigsten Kompetenzen überfachlicher Natur sind aus meiner Sicht die Fähigkeit, ein Team zielgenau zu entwi-
Führungskompetenzen für Leitende Ärzte -

Motivationsstrategien,

Teamführung und Kon-

fliktmanagement im

Krankenhaus

Hollmann, Jens

Springer-Verlag GmbH

1st Edition., 2010, XIII,

199 S. 30 Abb., Geb.

ckeln und zu führen, die Motivation und Leistungsbereitschaft der Mitarbeiter passgenau zu fördern sowie Konflikte souverän analysieren und konstruktiv bewältigen zu können.

\section{Wirken sich Führungskompetenzen karrierefördernd aus?}

- Auf jeden Fall; bei meinen Klienten kann ich überwiegend drei Interessenlagen erkennen: Die Klinikleitung will, dass ihre Ärzte kompetent führen und investiert in die Führungsausbildung ihrer Chefärzte; die Leitenden Ärzte bereiten sich mit der Führungsausbildung auf ihren nächsten Karriereschritt vor und die Chefärzte selbst festigen mit diesen Qualifikationen ihre Position und bauen sie aus.

Sie beraten und coachen Leitende Ärzte, welche thematischen Schwerpunkte sind entscheidend?

- Immer mehr ärztliche Führungspersönlichkeiten legen Wert auf ihre Kompetenzen in den Bereichen Teamführung, Motivationsstrategien und Konfliktmanagement. Es ist eine Erweiterung des Selbstverständnisses zu beobachten. Dort, wo die Klinikleitung noch nicht die Weitsicht hat, ihren Leitenden Ärzten ein solches Führungsprogramm zu ermöglichen, werden diese zunehmend selbst aktiv.

\section{Ihr abschließendes Statement?}

- Früher dachten viele Chefärzte, es ginge auch ohne qualifizierte und kompetente Führung. Natürlich geht es auch ohne, aber der nachhaltige Erfolg der Abteilung ist eben nicht sichergestellt oder wird sich sogar gar nicht erst einstellen.

- Weitere Infos www.medplus-kompetenz.de info@medplus-kompetenz.de

Das Interview führte Hinrich Küster, Springer Medizin Heidelberg 